\title{
Mapping of global, regional and national incidence, mortality and mortality-to-incidence ratio of lung cancer in 2020 and 2050
}

\author{
Rajesh Sharma ${ }^{1}$ iD
}

Received: 11 October 2021 / Accepted: 15 December 2021 / Published online: 12 January 2022

(c) The Author(s) under exclusive licence to Japan Society of Clinical Oncology 2022

\begin{abstract}
Aim Lung cancer is the leading cause of cancer deaths worldwide. This study examines the current and future burden of lung cancer at global, regional, and national levels.

Methods The estimates of lung cancer incident cases, deaths, and their age-standardized rates are drawn from GLOBOCAN 2020 for 21 regions and 185 countries. Mortality-to-incidence ratio (MIR) is considered as a proxy indicator of 5-year survival rates. Lung cancer burden in 2050 is projected using age-specific incidence and death rates in 2020.

Results In 2020, there were 2.21 million new cases and 1.8 million deaths due to lung cancer worldwide with age-standardized incidence rate (ASIR) of 22.4/100,000 (male: 31.5; female: 14.6) and age-standardized mortality rates (ASMR) of 18.0/100,000, (male: 25.9; female: 11.2/100,000). Global MIR of lung cancer was 0.82 (males 0.83; females: 0.79), varying from 0.59 (Japan) to 1.0 (Belize). Hungary had the highest age-standardized rates (ASIR: 50.1/100,000; ASMR: 42.4/100,000) and Nigeria (ASIR: 0.88; ASMR: 0.86) had the lowest age-standardized rates in 2020. Both ASIR and ASMR were positively correlated with country-level tobacco smoking prevalence and human development index (HDI), whereas MIR exhibited a negative correlation with HDI. As per our projections, there will be 3.8 million incident cases and 3.2 million deaths globally due to lung cancer in 2050 .

Conclusion With close to 2 million cases and deaths already in 2020, lung cancer has already become a global public health threat. Even with current risk levels and age-specific rates, lung cancer annual cases are expected to reach 3.8 million in 2050. Until smoking prevalence is reduced and ambient air pollution levels are checked, particularly in low/medium HDI countries, the lung cancer epidemic will continue unfolding.
\end{abstract}

Keywords Lung cancer $\cdot$ Incidence $\cdot$ Mortality $\cdot$ Epidemiology $\cdot$ GLOBOCAN

\section{Introduction}

Lung cancer is the second leading cancer in terms of new cases and the leading cancer in terms of cancer deaths worldwide [1]. From a rare disease about a 100 years ago [2], lung cancer has gained prominence as one of the leading cancers by the twenty-first century [1]. The growth in lung cancer burden has largely been attributed to the rise in cigarette smoking, which is expected to have peaked in industrialized countries (e.g., United States) by the later half of the twentieth century, after the landmark study by Doll and

Rajesh Sharma

rajesh.sharma@dtu.ac.in

1 University School of Management and Entrepreneurship, East Delhi Campus, Delhi Technological University, Room No. 305, Vivek Vihar Phase II, Delhi 110095, India
Hill [3] and United States surgeon general's report linking smoking as the causative factor of lung cancer [4]. Still, the tobacco smoking epidemic is unfolding in regions of Asia and Africa, due to which, the lung cancer burden is increasing in several countries of these regions [5]. Although lung cancer survival rates are one of the lowest among frequent cancer groups, there are wide disparities in 5-year survival rates across countries, from less than $10 \%$ in countries such as Brazil, Bulgaria, India, and Thailand to $32.9 \%$ in Japan [6].

About $80-85 \%$ of lung cancer is non-small cell lung cancer, which is further sub-divided into adenocarcinoma, squamous cell carcinoma, and large cell carcinoma [7] and $10-15 \%$ of lung cancer is small cell lung cancer and the rest is lung carcinoid tumor and other lung cancers [8]. About $8 \%$ of lung cancer is inherited or occur due to genetic predisposition $[9,10]$. Tobacco smoking is by far the single 
most significant risk factor of lung carcinoma [11], followed by air pollution (particularly particulate matter $\mathrm{PM}_{2.5}$ ) [12], passive smoking $[13,14]$, and occupational exposure to carcinogens such as radon, and asbestos [15]. The estimated economic cost of lung cancer to world economy was about $\$ 180$ billion in 2008 [16]. In the United States, the average treatment cost of lung cancer exceeds $\$ 45,000$ as compared to approximately $\$ 3,000$ in non-cancer patients [17]. As lung cancer is the leading cause of deaths and entails a huge economic burden globally, it is pertinent to understand the lung cancer burden such that interventions can be designed and implemented appropriately.

In this study, we provide a comprehensive and comparable examination of lung cancer epidemiology at global, regional, and national levels in 2020 using recently released GLOBOCAN 2020 estimates produced by International Agency for Research on Cancer [1, 18]. The lung cancer burden is evaluated using age, sex, and country-wise estimates of number of incident cases and deaths, age-standardized rates (per 100,000 population) and mortality-to-incidence ratio (MIR) in 185 countries. The human development index (HDI) is considered as the indicator of socio-economic status of countries. As tobacco smoking is the biggest risk factor of lung cancer, we also investigate the bivariate association between tobacco smoking prevalence and age-standardized rates of lung cancer. Lastly, we project the lung cancer incident cases and deaths to 2050 using the age-specific estimates of incidence and death rates in 2020 and age-specific population projections.

This comprehensive report on lung cancer epidemiology in the light of HDI and tobacco smoking prevalence is expected to aid our understanding of the lung cancer burden and help in policy formulation towards fighting lung cancer burden at the global, regional, and national levels.

\section{Data and Methods}

The age, sex, and country-specific estimates of lung cancer incidence and mortality in 185 countries for 2020 are procured from GLOBOCAN 2020 database [19]. The lung cancer burden described and examined in this study pertains to codes C33-34 (including trachea and bronchus) of the international classification of disease tenth version (ICD 10). The MIR for each country is calculated as the ratio between all-age death counts and all-age incident cases of lung cancer. The absolute counts (not the age-standardized rates) are provided with $95 \%$ uncertainty intervals. The age-specific counts and age-specific rates (per 100,000 populations) are available for eighteen 5-year non-overlapping age groups from below -5 to 85 -plus. The age-standardized rates are expressed per 100,000 population and are calculated using Segi's method $[1,18]$.
The estimates of lung cancer are available for 21 regions defined by the United Nations: Australia/New Zealand, Caribbean, Central America, Central, and Eastern Europe, Eastern Africa, Eastern Asia, Melanesia, Micronesia, Middle Africa, Northern Africa, Northern America, Northern Europe, Polynesia, South America, South-Central Asia, South-Eastern Asia, Southern Africa, Southern Europe, Western Africa, Western Asia, and Western Europe. Using age-specific rates of incidence and mortality in 21 regions in 2020 and age-specific population projections from the United Nations world population prospects (WPP) report, we project the lung cancer burden in 2050. The basic assumption underlying these 2050 projections is that the age-specific rates will remain unchanged by 2050 (i.e., the risk factors and the state of health care stays the same in each region). The equation for arriving at 2050 projection of incident cases is described below.

$I C_{2050}=\sum_{i=1}^{n} I R_{2020 i} \times P_{2050 i}$

In the above equation, $I C_{2050}$ denotes total number of incident cases in 2050, i denotes the age group, $I R_{2020 i}$ implies age group specific incidence rate in 2020 in the $\mathrm{i}^{\text {th }}$ age group and $P_{2050 i}$ denotes the projected population count in the $\mathrm{i}^{\text {th }}$ age group in 2050 as per WPP report by the United Nations. The estimates of number of deaths due to lung cancer in 2050 are also produced in the same manner. The HDI for 2019 represented a country's socio-economic status or development [20]. We also investigate the association between lung cancer age-standardized rates and tobacco smoking prevalence in countries. For this purpose, we employ Pearson's correlation coefficient to describe the bivariate association. The data on age-standardized tobacco smoking prevalence (\%) are procured from Global Health Observatory database of World Health Organization (WHO), which provided data for 149 countries and 6 WHO regions [Africa (32 countries), Americas (22), Eastern Mediterranean (15), Europe (48), South-East Asia (9), Western Pacific (23)] [21].

All the data analysis and data visualizations in this study are done using statistical software R 4.1.1, Stata 13.1, and Python 3.8.8.

\section{Results}

\section{Global burden of lung cancer in 2020}

Globally, there were 2.21 million [2.18-2.24 million] new cases and 1.8 million [1.77-1.83 million] deaths due to lung cancer in 2020. Males accounted for approximately twothirds of the global burden (1.44 million [1.41-1.46 million] new cases and 1.19 million [1.16-1.21 million] deaths). The 
global age-standardized incidence rate (ASIR) of lung cancer was $22.4 / 100,000$, with males ASIR $(31.5 / 100,000)$ being more than double that of females $(14.6 / 100,000)$. Global age-standardized mortality rates (ASMR) was 18.0/100,000, varying from $11.2 / 100,000$ in females to $25.9 / 100,000$ in males. The MIR at the global level was 0.82 with not much difference between males (0.83) and females (0.79).

\section{Lung cancer burden by region in 2020}

East Asia was the leading region in terms of incident cases and deaths with an estimated 1.01 million [1.00-1.02 million] incident cases and 841,174 [831,852-850,601] deaths in 2020, followed by Northern America (cases: 253,537 [252,452-254,627]; deaths: 159,641 [158,736-160,551]). Lung cancer was ranked among the top three cancer groups out of 34 cancer groups in terms of new cases in 11/21 regions and among top three in 15/21 regions in terms of cancer deaths (Table 1). The MIR of lung cancer varied from 0.63 in Northern America to 0.96 in Micronesia, with MIR being 0.80 or above in the majority of regions except Australia/New Zealand (0.69), Northern Europe (0.73) and Western Europe (0.78). Polynesia had the highest ASIR $(37.3 / 100,000)$ and Micronesia had the highest ASMR (34.9/100,000). Majority of African regions had low agestandardized rates, with Western Africa recording the lowest $\operatorname{ASIR}(2.2 / 100,000)$ and $\operatorname{ASMR}(2.1 / 100,000)$.

The male-female disparity in age-standardized rates was again visible across regions (Supplementary Fig. S1), with male age-standardized rates being higher than females in almost all regions. The male-female differences in agestandardized rates were starker in regions of Asia (Western Asia, South-East Asia) and Africa (Northern Africa, Southern Africa) than in Northern America, Northern Europe, Western Europe, and Australia/New Zealand (Supplementary Fig. S1).

\section{Lung cancer burden by country}

In absolute terms, China, United States, and Japan were the three leading countries with 815,563 [808,051-823,145], 227,875 [223,940-231,879], and $138,532[134,618-142,560]$ incident cases in 2020 , respectively; these three countries were also the leading countries in terms of death counts estimated at 714,699 [706,501-722,992], 138,225 [136,168-140,313], and 82,369 [80,835-83,932], respectively. In terms of agestandardized rates, Hungary was the leading country (ASIR:50.1/100,000 and ASMR:42.4/100,000), followed by Serbia (ASIR:47.3/100,000 and ASMR:40.0/100,000). Nigeria (ASIR: 0.88 and ASMR: 0.86) and Mozambique
(ASIR: 1.4 and ASMR: 1.4) had the lowest age-standardized rates among 185 countries in 2020 (Fig. 1).

\section{Age-specific incidence and mortality of lung cancer}

The lung cancer burden by age-groups illustrates that age groups below 45 years accounted for a very small percentage of lung cancer burden (2.4\% of incident cases and $1.9 \%$ of deaths). Both the metrics (incidence and deaths) peaked in the 65-69 years age group and declined thereafter (Fig. 2). The age-specific incidence and death rates rose with age such that the oldest age groups had the highest age-specific rates. To gauge the age-specific patterns of lung cancer burden in different regions, we aggregated the age-specific incident cases and deaths in four age groups: below 45 years, $45-59$ years, $60-69$ years, and $70-85$ plus and present the percentage of lung cancer burden in these age groups in Supplementary Fig. S2. In regions of Africa, a sizable fraction of incident cases occurred in age groups below 45 years (e.g., Western Africa 8.9\%, Eastern Africa $10.4 \%$, Middle Africa $11.1 \%$ ), whereas only $1-2 \%$ of lung cancer cases occurred in age groups below 45 years in Europe (Central and Eastern Europe 1.6\%, Western Europe $1.0 \%$, Southern Europe 1.0\%). In regions such as Western Africa and South-Eastern Asia, around $60 \%$ of cases and deaths occurred in age groups 60 years or older, whereas in Western Europe and South America, around $80 \%$ of new cases and deaths occurred in age groups 60 years or older. In Western Europe, Southern Europe, Northern Europe, and Australia/New Zealand, more than 50\% of incident cases and deaths occurred in age groups 70 years or older, while in regions of Africa (Western Africa, Middle Africa, and Eastern Africa), approximately $30 \%$ of lung cancer burden occurred in age groups 70 years or older (Supplementary Fig. S2).

\section{Bivariate relationship between HDI and lung cancer burden}

Figure 3 displays the bivariate associations between country-level age-standardized rates and MIR with the socioeconomic development status of countries measured by HDI. It shows that both ASIR and ASMR were positively associated with HDI with pairwise correlation coefficient of 0.75 and 0.70 , respectively; on the other hand, MIR was negatively correlated with country-level HDI $(r=-0.67)$. This implies that, on average, countries with higher HDI had higher age-standardized rates (Fig. 3a, b); however, more developed countries (countries with higher HDI) had lower MIR (i.e., higher 5-year survival rates) than lesser developed ones. For instance, high HDI countries such as Japan (0.59), United States (0.61), and Australia 
Table 1 Lung cancer burden by region in 2020

\begin{tabular}{|c|c|c|c|c|c|}
\hline Population & Incidence & ASIR & Deaths & ASMR & MIR \\
\hline Australia and New Zealand & $\begin{array}{l}15,587 \\
{[15305-15874]} \\
{[[5]]}\end{array}$ & 25.2 & $\begin{array}{l}10,791 \\
{[10550-11038]} \\
{[[1]]}\end{array}$ & 16.2 & 0.69 \\
\hline Caribbean & $\begin{array}{l}11,058 \\
{[10485-11662]} \\
{[[4]]}\end{array}$ & 17.6 & $\begin{array}{l}10,079 \\
{[9604-10578]} \\
{[[6]]}\end{array}$ & 15.8 & 0.91 \\
\hline Central America & $\begin{array}{l}9934 \\
{[9615-10264]} \\
{[[9]]}\end{array}$ & 5.2 & $\begin{array}{l}9236 \\
{[8979-9501]} \\
{[[6]]}\end{array}$ & 4.8 & 0.93 \\
\hline Central and Eastern Europe & $\begin{array}{l}151,632 \\
{[149886-153398]} \\
{[[3]]}\end{array}$ & 26.9 & $\begin{array}{l}130,596 \\
{[129152-132056]} \\
{[[1]]}\end{array}$ & 22.7 & 0.86 \\
\hline Eastern Africa & $\begin{array}{l}7419 \\
{[5268-10448]} \\
{[[11]]}\end{array}$ & 3.5 & $\begin{array}{l}6758 \\
{[4577-9978]} \\
{[[11]]}\end{array}$ & 3.2 & 0.91 \\
\hline Eastern Asia & $\begin{array}{l}1,012,021 \\
{[1003100-1021020]} \\
{[[1]]}\end{array}$ & 34.4 & $\begin{array}{l}841,174 \\
{[831852-850601]} \\
{[[1]]}\end{array}$ & 28.1 & 0.83 \\
\hline Melanesia & $\begin{array}{l}918 \\
{[768-1097]} \\
{[[6]}\end{array}$ & 13.1 & $\begin{array}{l}798 \\
{[736-865]} \\
{[[4]]}\end{array}$ & 11.5 & 0.87 \\
\hline Micronesia & $\begin{array}{l}202 \\
{[152-269]} \\
{[[1]]}\end{array}$ & 36.4 & $\begin{array}{l}194 \\
{[146-258]} \\
{[[1]]}\end{array}$ & 34.9 & 0.96 \\
\hline Middle Africa & $\begin{array}{l}2037 \\
{[1382-3003]} \\
{[[12]]}\end{array}$ & 2.5 & $\begin{array}{l}1897 \\
{[1287-2795]} \\
{[[10]]}\end{array}$ & 2.4 & 0.93 \\
\hline Northern Africa & $\begin{array}{l}23,179 \\
{[20865-25750]} \\
{[[3]]}\end{array}$ & 11.1 & $\begin{array}{l}20,728 \\
{[18443-23296]} \\
{[[3]]}\end{array}$ & 9.9 & 0.89 \\
\hline Northern America & $\begin{array}{l}253,537 \\
{[252452-254627]} \\
{[[2]]}\end{array}$ & 32.6 & $\begin{array}{l}159,641 \\
{[158736-160551]} \\
{[[1]]}\end{array}$ & 19.3 & 0.63 \\
\hline Northern Europe & $\begin{array}{l}75,051 \\
{[74261-75849]} \\
{[[2]]}\end{array}$ & 29.7 & $\begin{array}{l}54,421 \\
{[53732-55119]} \\
{[[1]]}\end{array}$ & 20.1 & 0.73 \\
\hline Polynesia & $\begin{array}{l}268 \\
{[212-339]} \\
{[[1]]}\end{array}$ & 37.3 & $\begin{array}{l}229 \\
{[159-330]} \\
{[[1]]}\end{array}$ & 31.8 & 0.85 \\
\hline South America & $\begin{array}{l}76,609 \\
{[72588-80853]} \\
{[[4]]}\end{array}$ & 13.6 & $\begin{array}{l}67,312 \\
{[66320-68319]} \\
{[[1]]}\end{array}$ & 11.8 & 0.88 \\
\hline South-Central Asia & $\begin{array}{l}121,369 \\
{[116397-126554]} \\
{[[4]]}\end{array}$ & 6.6 & $\begin{array}{l}109,356 \\
{[104882-114021]} \\
{[[2]]}\end{array}$ & 5.9 & 0.9 \\
\hline South-Eastern Asia & $\begin{array}{l}123,309 \\
{[118258-128576]} \\
{[[2]]}\end{array}$ & 17.2 & $\begin{array}{l}109,520 \\
{[104946-114294]} \\
{[[1]]}\end{array}$ & 15.3 & 0.89 \\
\hline Southern Africa & $\begin{array}{l}9178 \\
{[8893-9473]} \\
{[[4]]}\end{array}$ & 16.9 & $\begin{array}{l}7939 \\
{[7707-8178]} \\
{[[1]]}\end{array}$ & 14.6 & 0.87 \\
\hline Southern Europe & $\begin{array}{l}104,391 \\
{[101664-107191]} \\
{[[3]]}\end{array}$ & 28.7 & $\begin{array}{l}85,635 \\
{[84449-86838]} \\
{[[1]]}\end{array}$ & 21.9 & 0.82 \\
\hline Western Africa & $\begin{array}{l}4175 \\
{[2688-6485]} \\
{[[11]]}\end{array}$ & 2.2 & $\begin{array}{l}3849 \\
{[3370-4396]} \\
{[[10]]}\end{array}$ & 2.1 & 0.92 \\
\hline
\end{tabular}


Table 1 (continued)

\begin{tabular}{llllll}
\hline Population & Incidence & ASIR & Deaths & ASMR & MIR \\
\hline Western Asia & 58,437 & 24.2 & 52,467 & 21.9 & 0.9 \\
& {$[54808-62307]$} & & {$[51245-53718]$} & & \\
Western Europe & {$[[2]]$} & & {$[[1]]$} & & \\
& 146,460 & 32.7 & 113,524 & 23.8 & 0.78 \\
& {$[144672-148270]$} & & {$[112268-114794]$} & & \\
\hline
\end{tabular}

The MIR is calculated as the ratio between all-age death counts and all-age cases. The numbers in the parenthesis represent $95 \%$ uncertainty interval. The number in double parenthesis denotes the rank of lung cancer among 34 cancer groups in that region. Data source: GLOBOCAN 2020 (International Agency for Research on Cancer)

Incidence incident cases, ASIR age-standardized incidence rate, Deaths number of lung cancer deaths, $A S M R$ age-standardized mortality rate; MIR mortality-to-incidence ratio

(0.67) had much lower MIR than countries such as Belize, Comoros, and Vanuatu, which had MIR of 1.0 (Fig. 3c).

\section{Smoking and lung cancer}

Worldwide, tobacco smoking prevalence was $21.9 \%$ in 2016 , varying from $4 \%$ in Ghana to $47.4 \%$ in Kiribati (Fig. 4) with tobacco smoking prevalence exceeding $25 \%$ in 57/149 countries. The tobacco smoking prevalence was high in European countries with 5 of the top-10 countries among 149 countries belonged to Europe. Notably, 11/33 countries in Africa had a smoking prevalence less than $10 \%$, whereas all countries in Europe had a smoking prevalence greater than $10 \%$. Next, we explore the bivariate relationship between age-standardized rates of lung cancer and tobacco smoking prevalence (Supplementary Fig. S3). Tobacco smoking prevalence was positively associated with ASIR $(r=0.61)$ and ASMR ( $r=0.64$ ) (Supplementary Fig. S3a, b). It implies that countries with higher smoking prevalence rates also had higher age-standardized rates of lung cancer.

\section{Lung cancer burden in 2050}

In 2050, the global burden of lung cancer is projected to reach 3.8 million new cases and 3.2 million deaths each year. In 2050, lung cancer cases and deaths will be more than 100,000 in 10/21 regions led by Eastern Asia, which is projected to record 1.7 million incident cases and 1.5 million deaths (Fig. 5). The burden of lung cancer in regions of Asia (South-Eastern Asia, South-Central Asia, and Western Asia) and Africa (Northern Africa, Southern Africa, and Western Africa) is expected to double or more between 2020 and 2050, whereas regions in Europe (Northern Europe, Southern Europe, Western Europe, Central and Eastern Europe) are expected to witness the smallest increases in the lung cancer burden (Fig. 5). As a result of these changes, the lung cancer burden in several Asian regions will surpass that in a few regions of Europe. For instance, South-Eastern Asia $(123,309)$ and South-Central Asia $(121,369)$ had lesser number of incident cases than Central and Eastern Europe $(151,632)$ and Western Europe $(146,460)$ in 2020; however, by 2050, these two regions in Asia will surpass the number of incident cases in Central and Eastern Europe and Western Europe. In 2050, lung cancer is projected to claim similar number of lives in South-Eastern Asia $(248,326)$ and South-Central Asia $(238,020)$ as that in Northern America $(253,058)$, although the projected number of incident cases in Northern America $(378,587)$ is much higher than projected new cases in South-Eastern Asia $(271,416)$ and South-Central Asia (264,309) (Fig. 5).

\section{Discussion}

With close to 2 million incident cases and deaths along with an MIR of 0.82 in 2020, lung cancer is one of the most lethal neoplasms and was ranked first among 34 cancer groups in 13/21 regions in terms of cancer-related deaths in 2020 . The age-standardized rates were the highest in regions of Europe and the Americas, where smoking prevalence is still higher than that in Africa and Asia. Due to lower smoking prevalence and lower life expectancy in sub-Saharan Africa (SSA), the age-standardized rates, as well as the absolute burden of lung cancer, is low; as a result, lung cancer, which is currently ranked first in terms of cancer deaths in regions of Europe and America, is ranked much behind other neoplasms in SSA. Country-wise, China, United States, and Japan are the leading countries, accounting for $53.6 \%$ of incident cases and $52.1 \%$ of lung cancer deaths.

The age-standardized rates of lung cancer displays positive correlation with HDI as well as tobacco smoking prevalence, which implies that more developed countries in Europe, America, Oceania have higher smoking prevalence (also clear from Fig. 4 on smoking prevalence) as well as higher age-standardized rates of lung cancer. In Europe, the rates of lung cancer are decreasing among males in the majority of countries, whereas the lung cancer rates are rising or plateauing among females in several countries and 


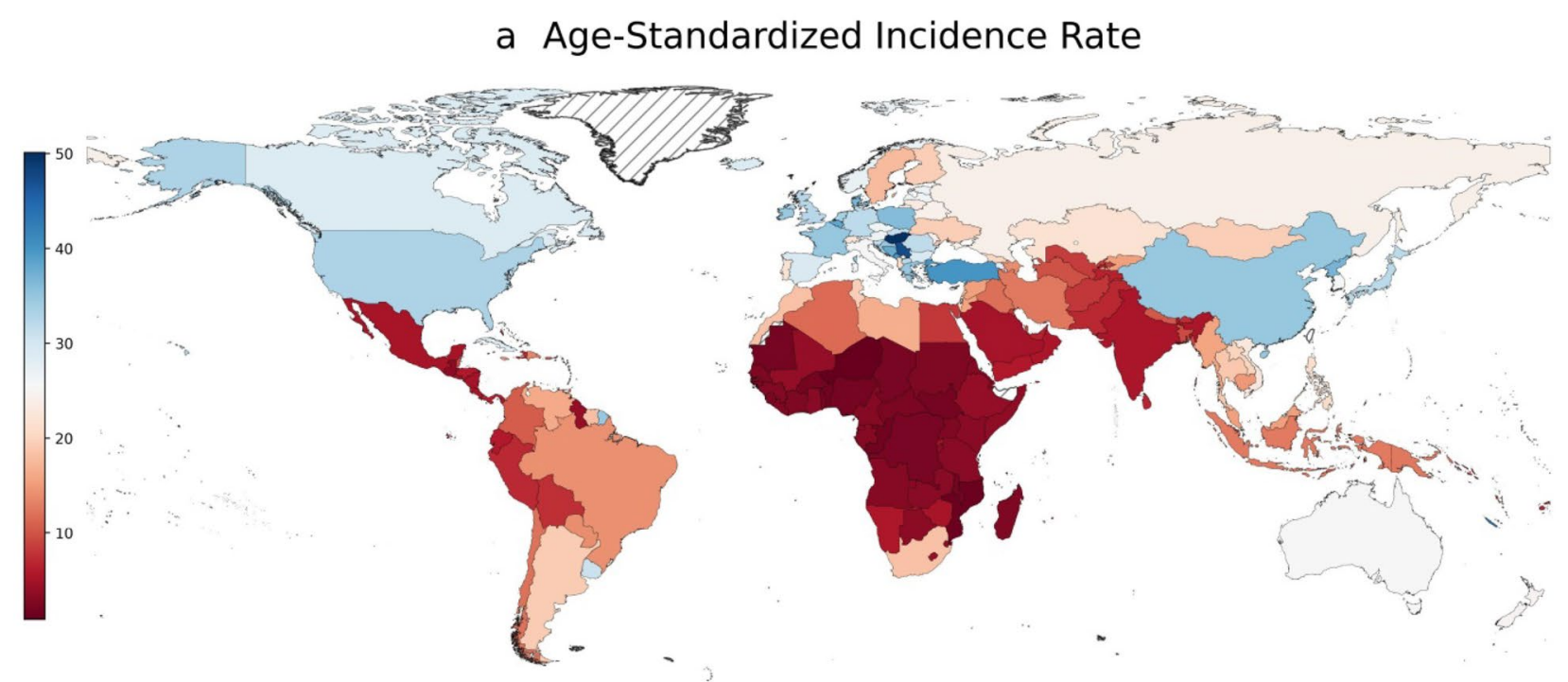

b Age-Standardized Mortality Rate

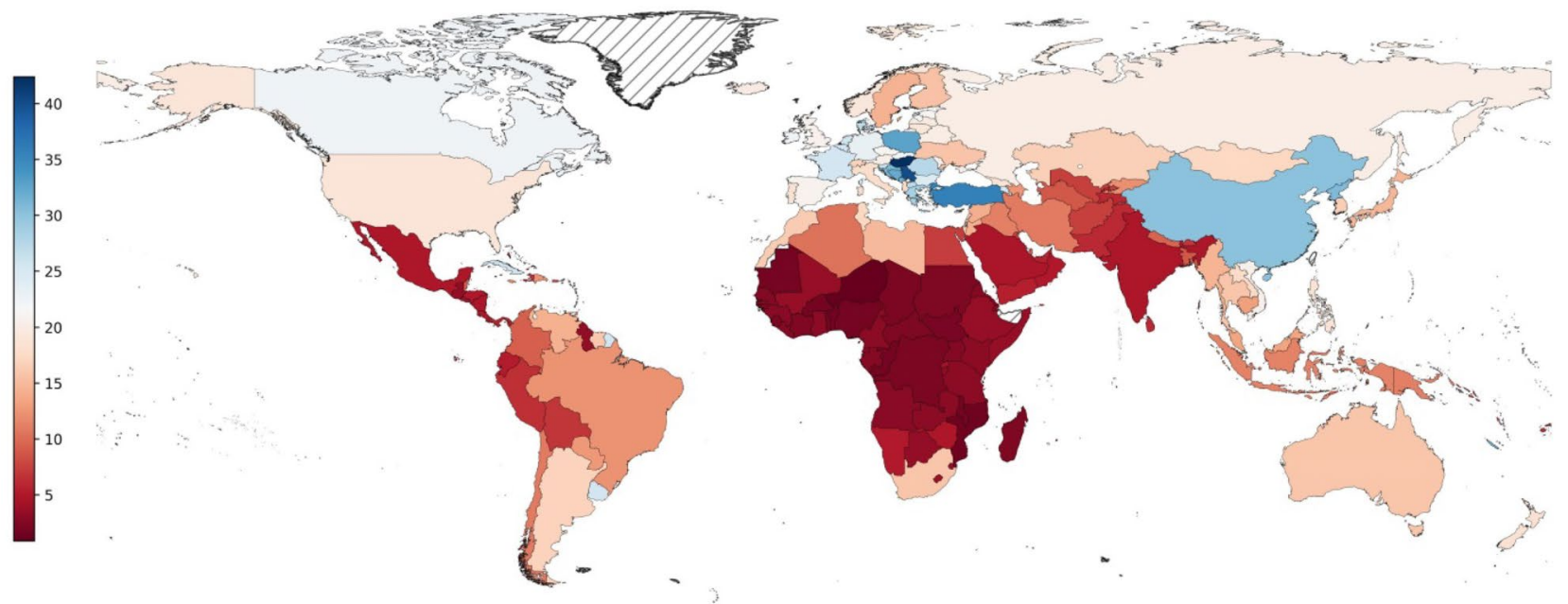

c Mortality-to-Incidence Ratio

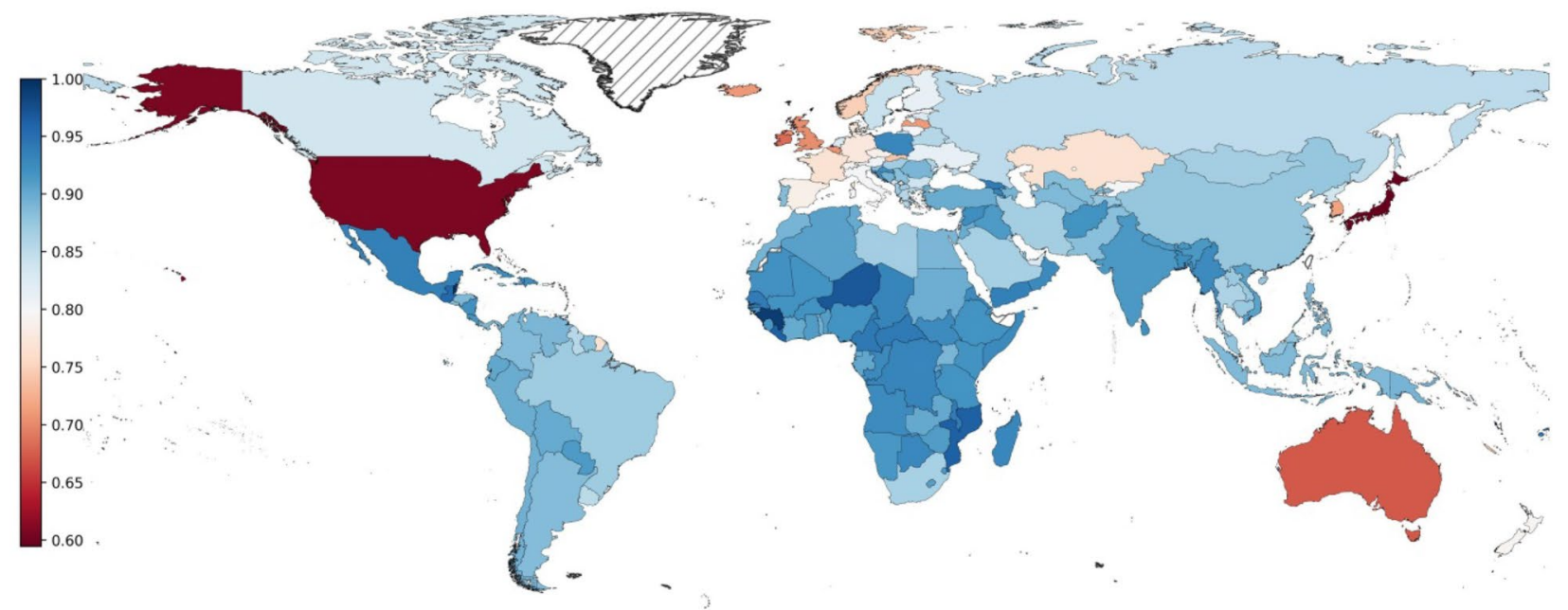


4Fig. 1 Geographical pattern of age-standardized rates of lung cancer in 2020. The mortality-to-incidence ratio is calculated as the ratio between all-age death counts and all-age cases. The age-standardized rates are expressed in terms of counts per 100,000 population. Data source: GLOBOCAN 2020 (International Agency for Research on Cancer)

these patterns most likely reflect the differing patterns of the smoking epidemic among males and females [22].

Although MIR is negatively associated with HDI, yet the 5-year survival rates of lung cancer are one of the lowest (i.e., high MIR) among all cancers, even in the high HDI countries. For instance, the 5-year survival rate of lung cancer is $19 \%$ in the United States, one of the lowest among the most frequent cancer groups [23]. Low survival rates of lung cancer also reflect a higher percentage of cases detected in the metastatic phase, which has much lower survival rate (5\%) than localized lung cancer (57\%) [23]. Low-dose computed tomography (LDCT) has been recently suggested as a screening solution for lung cancer. The LDCT has been shown to reduce lung cancer mortality in both National Lung Screening Trial and Multicentric Italian Lung Detection trials [24, 25]. For select current/former heavy smokers, the
American Cancer Society and US Preventive Services Task Force have recommended LDCT screening [23]. However, there are questions on LDCT screening in terms of high false-positive rates [24, 26-28], overdiagnosis [24], large percentage of tumors detected in advanced stages [26] and risk of radiation-induced neoplasms [29]. Moreover, the LDCT screening becomes more challenging in low-resource countries as healthcare workers' expertise in adjusting radiation dose is low and secondly, LDCT screening is expected to cost $\$ 81,000$ per one quality-adjusted life-year gained [30].

Currently, countries in Africa have very low incidence and mortality rates barring a few countries in Northern and Southern Africa. These patterns also follow the low smoking prevalence rates in Africa, barring a few countries in Northern Africa (e.g., Algeria 15.6\%) and Southern Africa (e.g., Lesotho 27.2\%; South Africa 20.7\%; Botswana: 20.1\%). However, in persons living with HIV/AIDS, smoking rates are 2-4 times higher than the general population in Africa [31]. The lung cancer burden in Africa is expected to double or more by 2050 based on demographic changes alone. As smoking initiation before the age of 18 years significantly predicts smoking in adult age (and hence the elevated lung

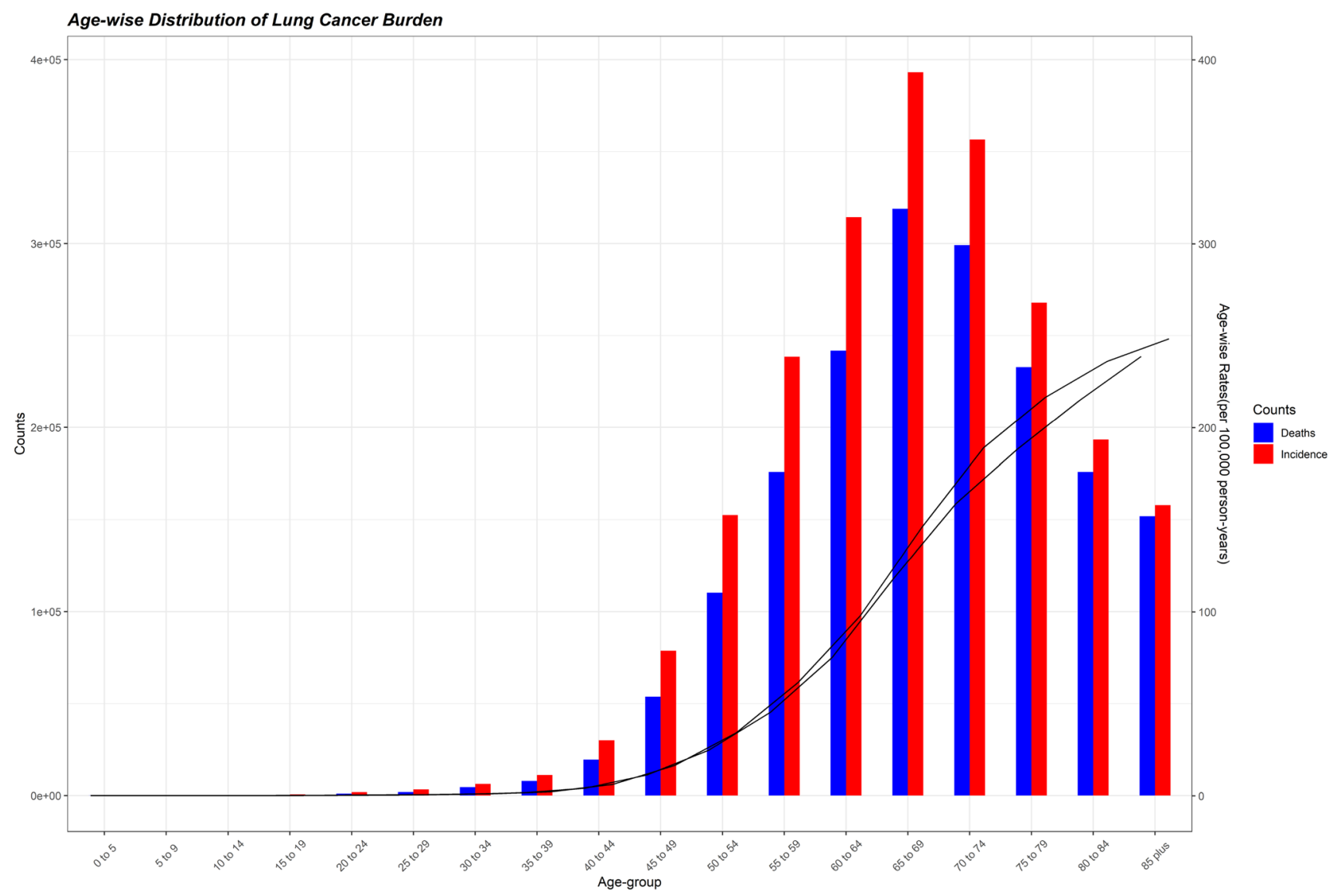

Fig. 2 Age-specific burden of lung cancer in 2020. Deaths: age-specific death count; incidence: age-specific incident cases. Data source: GLOBOCAN 2020 (International Agency for Research on Cancer) 


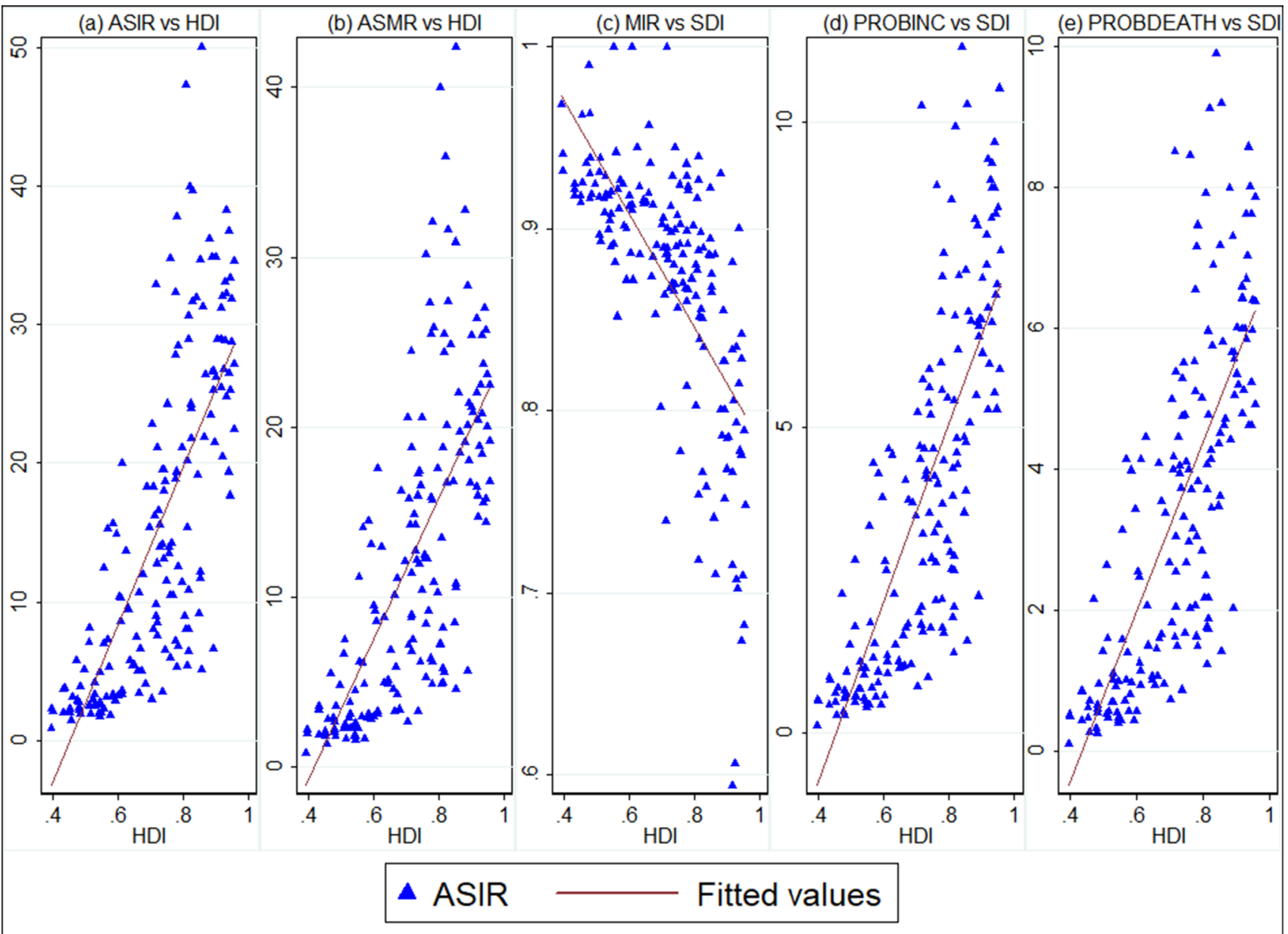

Fig. 3 Bivariate relationship between age-standardized rates, MIR of lung cancer and HDI. ASIR age-standardized incidence rate; $A S M R$ age-standardized mortality rate; MIR mortality-to-incidence ratio; $H D I$ human development index. MIR was calculated as the ratio between all-age death counts and all-age incident cases. Data source of cancer estimates: GLOBOCAN 2020 (International Agency for Research on Cancer). The data pertaining to HDI are procured from United Nations Development Program

\section{Tobacco Smoking Prevalence (\%)}

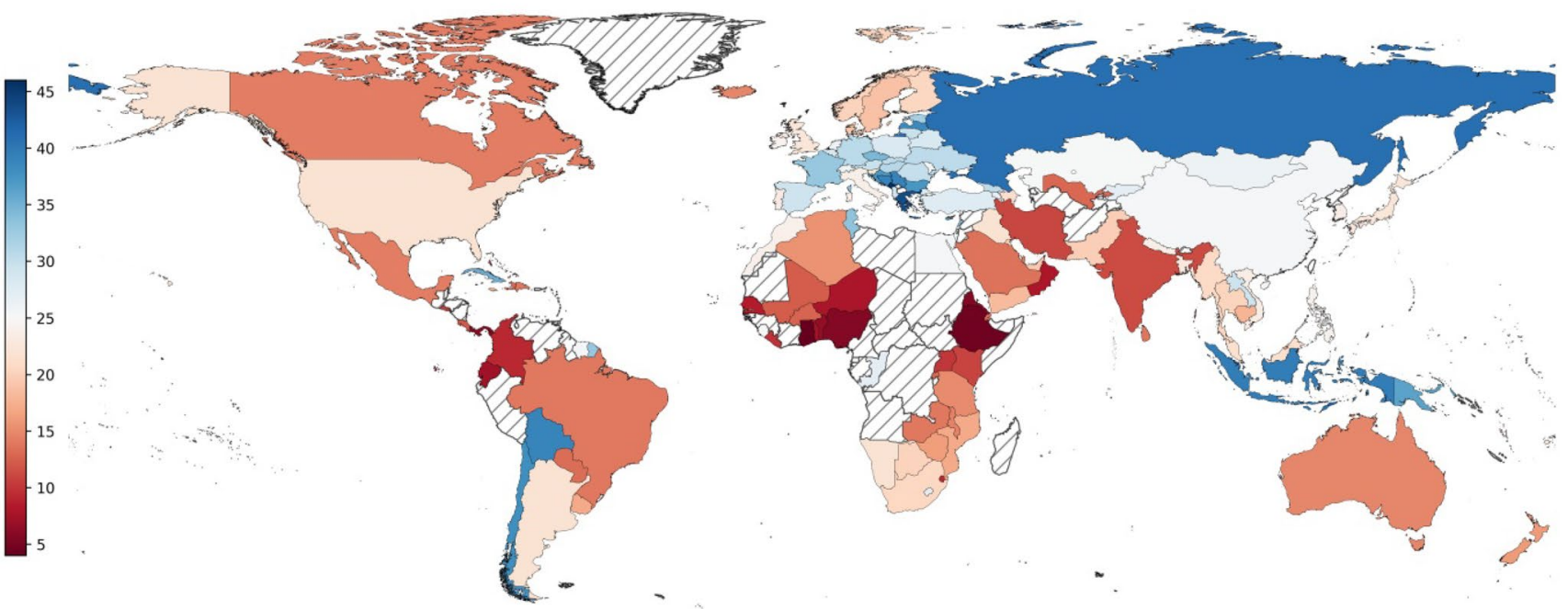

Fig. 4 Geographical pattern of tobacco smoking prevalence. Tobacco smoking prevalence (\%) pertains to the year 2016. Data source: World Health Organization (WHO) 


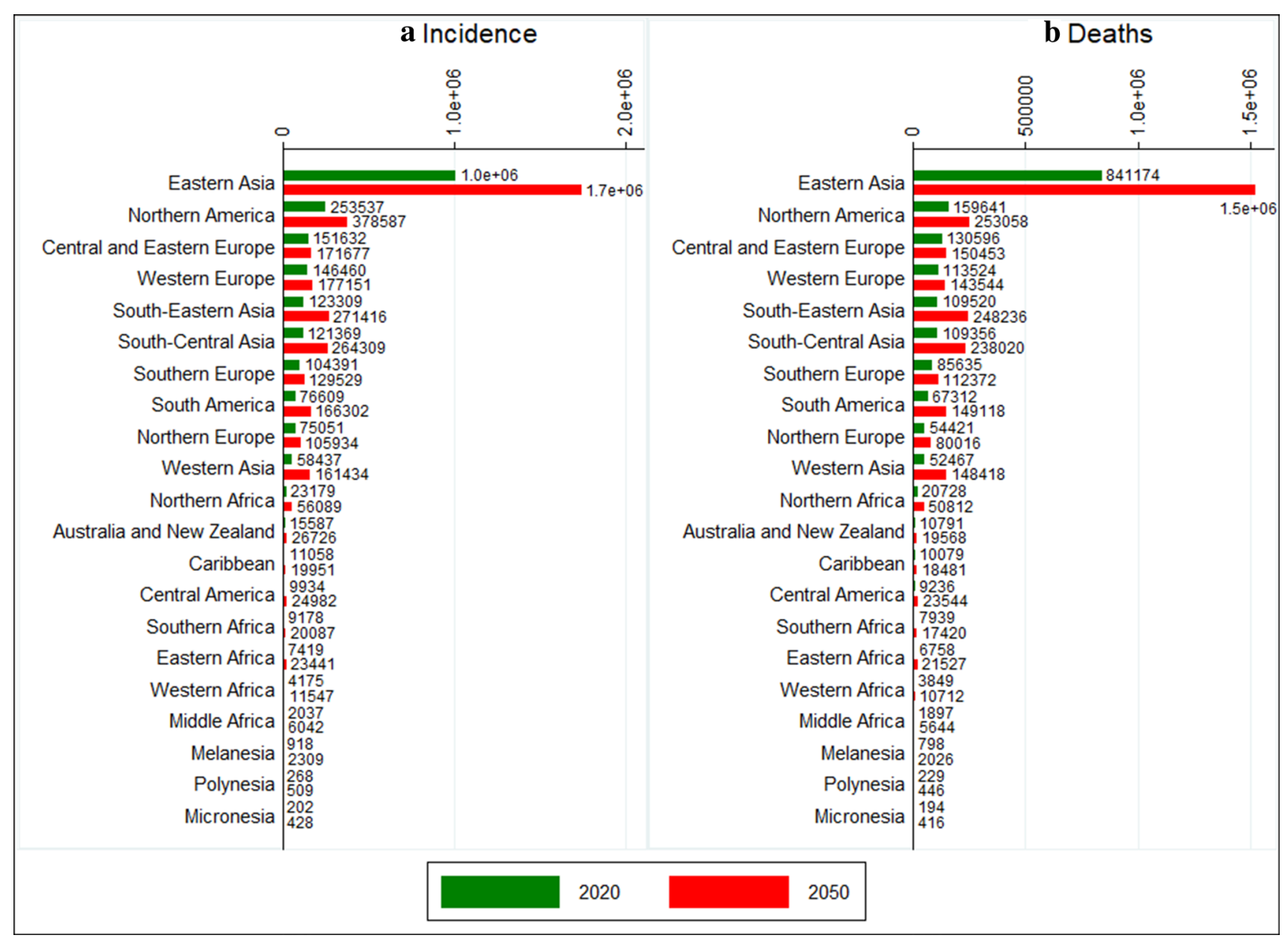

Fig. 5 Region wise projected incidence and deaths of lung cancer in 2050. Incidence: all-age incident cases; deaths: all-age deaths. Data source: authors' calculation based on age-specific rates of lung cancer as per GLOBOCAN 2020 estimates

cancer risk), a study found smoking prevalence as high as $9.6 \%$ in Cote d'Ivoire among 12/13 year old [32]. Moreover, air pollution levels are also rising in Africa. As per the State of Global Air 2020 report [33], 5/10 countries with the worst annual average $\mathrm{PM}_{2.5}$ concentrations belonged to Africa (Niger: 3rd, Nigeria: 5th; Egypt: 6th; Mauritiania: 7th; Cameroon: 8th). If these trends of early smoking initiation and ambient air pollution levels continue along with rising life expectancy, the lung cancer burden in Africa is expected to be even higher in 2050 than our projections.

Even though Asia is in the initial stages of the smoking epidemic [34-37], the continent already accounted for $60 \%$ of incident cases and $62 \%$ of all lung cancer deaths in 2020 . If current rates persist in the future, there will be 2.4 million new cases and 2.2 million deaths in Asia due to lung cancer in 2050. Previous research has also found that while lung cancer burden has been decreasing in the industrialized countires after a peak in the 2000s, it is gradually increasing in China [38]. A recent study has found that smoking prevalence has increased among males in China and India and plateaued in Japan, South Korea, Singapore, and Taiwan [37]. Moreover, the mean age of smoking initiation has decreased in successive birth cohorts, and the mean number of cigarettes smoked per day has increased [37]. Therefore, anti-smoking campaigns and public awareness campaigns about health risks of smoking are urgently required to stop and reverse the smoking epidemic in Asia.

The absolute counts as well as age-standardized rates of lung cancer are consistently higher in males than females across regions. Higher proportion of lung cancer burden in males is attributed to differences in smoking prevalence, with $80 \%$ of males and $50 \%$ of females with lung cancer are/were smokers [7]. However, male-female differences in lung cancer burden cannot be fully explained by differences in smoking prevalence. As many as one-third of lung cancer patients in East Asia are non-smokers, most of whom present with lung adenocarcinoma [39]. Even in industrialized countries, high lung cancer incidence rate in women born around 1960 cannot be fully explained by increasing smoking incidence rates in women [40]. Second-hand smoke at home and 
workplace and cooking oil fumes are implicated as a few of the risk factors apart from other factors (genetic susceptibility, occupational exposure, hormonal status, and pre-existing lung disease) for rising lung cancer in never-smokers in East Asia [39]. Apart from tobacco smoking (personal or passive), air pollution is another significant risk factor of lung cancer [41], particularly among non-smokers [42, 43]. A meta-analysis of the relationship between $\mathrm{PM}_{2.5}$ and lung cancer burden (incidence and deaths) found a higher risk of both lung cancer incidence and death due to higher levels of $\mathrm{PM}_{2.5}$ levels, irrespective of smoking status and gender [44].

\section{Limitations}

As with all studies, this study also has some limitations. First, the lung cancer estimates of several countries are derived either from regional cancer registries or from neighboring countries due to the absence of data from highquality population-based cancer registries by the GLOBOCAN $[1,18]$. Therefore, in the countries lacking PBCRs, the actual estimates are likely to be different from the estimates reported in this study. Second, the 2020 estimates by GLOBOCAN do not reflect the effect of the COVID-19 pandemic. Therefore, actual cancer-related incident cases and deaths might be different as health services to non-COVID patients have been severely disrupted across the globe. Third, the 2050 projections reflect the demographic changes in population composition but do not reflect changes in risk factors (e.g., smoking, air pollution, among others) and improvements in cancer registration and health care delivery, which may occur in developing countries of Asia and Africa. As a result, due to worsening smoking and air pollution levels in developing countries of Asia and Africa, the lung cancer burden is expected to be much higher than our projections.

\section{Conclusion}

Lung cancer incident cases and deaths are projected to reach 3.8 million and 3.2 million, by 2050 based on demographic changes alone. Although age-standardized rates are the highest in high HDI countries of Europe and America, possibly on account of high smoking prevalence; the absolute burden, however, is the largest in developing countries led by China. With worsening smoking prevalence and $\mathrm{PM}_{2.5}$ levels in countries such as China, India, and Indonesia; Asia is expected to remain the epicenter of the lung cancer epidemic. As Africa is experiencing increases in smoking incidence, rising life expectancy, and pollution levels, the lung cancer burden is expected to be much higher in Africa by 2050 than our projections. The experience of highincome countries has taught that unless smoking patterns are reversed, the lung cancer cases and deaths are expected to grow unabated in developing countries. Additionally, countering the burden of lung cancer also requires curtailment of other risk factors such as air pollution and exposure to carcinogens.

Supplementary Information The online version contains supplementary material available at https://doi.org/10.1007/s10147-021-02108-2.

Funding This research received no specific grant from any funding agency in the public, commercial, or not-for-profit sectors.

Availability of data and material All the data are available on https:// gco.iarc.fr/today/online-analysis-table.

\section{Declarations}

Conflict of interest The authors declare that they have no conflict of interest.

Ethical approval and consent to participate The research was conducted using data available in the public domain and did not include any human participants or animals. Therefore, no ethical approvals were required.

Consent for publication The authors of the study have consent and responsibility for submission to the journal.

Patient consent statement Not Applicable.

Permission to reproduce material from other sources All the data used in this study are available in public domain. The geographical maps in the manuscript are drawn using GADM in Python and does not reflect author's views on geographical boundaries of the represented countries.

\section{References}

1. Sung H, Ferlay J, Siegel RL et al (2021) Global cancer statistics 2020: GLOBOCAN estimates of incidence and mortality worldwide for 36 cancers in 185 countries. CA Cancer J Clin 71(3):209-249

2. Adler I (1912) Primary malignant growths of the lungs and bronchi: A pathological and clinical study. Longmans, Green.

3. Doll R, Hill AB (1954) The mortality of doctors in relation to their smoking habits. BMJ 1(4877): 1451

4. Terry LL (1964) Washington, DC: U.S. Department of Health, Education, and Welfare; 1964. Smoking and Health: Report of the Advisory Committee to the Surgeon General of the Public Health Service. In: U-23 U.S. Department of Health, Education, and Welfare. Public Health Service; Publication No. 1103

5. Deng Y, Zhao P, Zhou L et al (2020) Epidemiological trends of tracheal, bronchus, and lung cancer at the global, regional, and national levels: a population-based study. J Hematol Oncol 13:98

6. Allemani C, Matsuda T, Carlo V et al (2018) Global surveillance of trends in cancer survival 2000-14 (CONCORD-3): analysis of individual records for 37513025 patients diagnosed with one of 18 cancers from 322 population-based registries in 71 countries. Lancet 391(10125):1023-1075 
7. Gridelli C, Rossi A, Carbone D et al (2015) Non-small-cell lung cancer. Nat Rev Dis Primers 1:15009

8. American Cancer Society (2021). https://www.cancer.org/cancer/lung-cancer/about/what-is.html. Accessed 15 June 2021

9. Vikis HG, Wang D, Lu Y et al (2008) Familial aggregation of common sequence variants on $15 q 24-251$ in lung cancer. J Natl Cancer Inst 100:1326-1330

10. Jonsson S, Thorsteinsdottir U, Gudbjartsson DF et al (2004) Familial risk of lung carcinoma in the icelandic population. JAMA 292:2977-2983

11. Sasco AJ, Secretan MB, Straif K (2004) Tobacco smoking and cancer: a brief review of recent epidemiological evidence. Lung Cancer 45:S3-9

12. Turner MC, Andersen ZJ, Baccarelli A et al (2020) Outdoor air pollution and cancer: An overview of the current evidence and public health recommendations. CA Cancer J Clin 70(6):460-479

13. International Agency for Research on Cancer (2002) Tobacco smoke and involuntary smoking. IARC, Lyon

14. Office on Smoking and Health (US) (2006) The health consequences of involuntary exposure to tobacco smoke: a report of the surgeon general [internet]. Centers for Disease Control and Prevention, Atlanta

15. de Groot PM, Wu CC, Carter BW et al (2018) The epidemiology of lung cancer. Transl Lung Cancer Res 7(3):220

16. American Cancer Society (ACS) (2021) The Global Economic Cost of Cancer. http://phrma-docs.phrma.org/sites/default/files/ pdf/08-17-2010_economic_impact_study.pdf. Accessed 10 Oct 2021

17. Kutikova L, Bowman L, Chang S et al (2005) The economic burden of lung cancer and the associated costs of treatment failure in the United States. Lung Cancer 50(2):143-154

18. Ferlay J, Colombet M, Soerjomataram I et al (2020) Cancer statistics for the year 2020: an overview. Int J Cancer. https://doi.org/ 10.1002/ijc. 33588

19. Ferlay J, Ervik M, Lam F et al (2020) Global cancer observatory: cancer today. International Agency for Research on Cancer, Lyon

20. United Nations Development Program (2020) Human development database. http://hdr.undp.org/en/data\#. Accessed 7 Apr 2021

21. World Health Organisation (2021). https://apps.who.int/gho/data/ node.sdg.3-a-viz?lang=en. Accessed 16 Jun 2021

22. Bray FI, Weiderpass E (2010) Lung cancer mortality trends in 36 European countries: secular trends and birth cohort patterns by sex and region 1970-2007. Int J Cancer 126(6):1454-1466

23. Siegel RL, Miller KD, Jemal A (2020) Cancer statistics, 2020. CA A Cancer J Clin 70:7-30. https://doi.org/10.3322/caac.21590

24. National Lung Screening Trial Research Team, Aberle DR, Adams AM et al (2011) Reduced lung-cancer mortality with low-dose computed tomographic screening. N Engl J Med 365:395-409

25. Pastorino U, Silva M, Sestini S et al (2019) Prolonged lung cancer screening reduced 10-year mortality in the MILD trial: new confirmation of lung cancer screening efficacy. Ann Oncol 30:1162-1169

26. Ruano-Ravina A, Heleno B, Fernández-Villar A (2015) Lung cancer screening with low-dose CT (LDCT), or when a public health intervention is beyond the patient's benefit. J Epidemiol Commun Health 69:99-100

27. Kramer BS, Berg CD, Aberle DR et al (2011) Lung cancer screening with low-dose helical CT: results from the National Lung Screening Trial (NLST). J Med Screen 18:109-111
28. Patz EF et al (2014) Overdiagnosis in low-dose computed tomography screening for lung cancer. JAMA Intern Med 174:269-274

29. Moyer VA (2014) Screening for lung cancer: US Preventive Services Task Force recommendation statement. Ann Intern Med 160:330-338

30. Black WC, Gareen IF, Soneji SS et al (2014) Cost-effectiveness of CT screening in the National Lung Screening Trial. N Engl J Med 371:1793-1802

31. Peprah E, Armstrong-Hough M, Cook SH et al (2021) An emerging syndemic of smoking and cardiopulmonary diseases in people living with HIV in Africa. Int J Environ Res Public Health 18(6):3111

32. Veeranki SP, John RM, Ibrahim A et al (2017) Age of smoking initiation among adolescents in Africa. Int J Public Health 62(1):63-72

33. Health Effects Institute (2020) State of Global Air 2020. Data source: Global Burden of Disease Study 2019. IHME, Washington

34. Clow B, Pederson A, Haworth-Brockman M et al (2009) Rising to the challenge: sex- and gender-based analysis for health planning, policy and research in Canada. Atlantic Centre of Excellence for Women's Health, Halifax

35. Lopez AD, Collishaw NE, Piha T (1994) A descriptive model of the cigarette epidemic in developed countries. Tob Control 3(3):242-247. https://doi.org/10.1136/tc.3.3.242

36. Zheng W, McLerran DF, Rolland BA et al (2014) Burden of total and cause-specific mortality related to tobacco smoking among adults aged $\geq 45$ years in Asia: a pooled analysis of 21 cohorts. PLoS Med 11(4):e1001631

37. Yang JJ, Yu D, Wen W et al (2019) Tobacco smoking and mortality in Asia: a pooled meta-analysis. JAMA Netw Open 2(3):e191474

38. Yang D, Liu Y, Bai C et al (2020) Epidemiology of lung cancer and lung cancer screening programs in China and the United States. Cancer Lett 468:82-87

39. Zhou F, Zhou C (2018) Lung cancer in never smokers-the East Asian experience. Transl Lung Cancer Res 7(4):450

40. Jemal A, Miller KD, Ma J et al (2018) Higher lung cancer incidence in young women than young men in the United States. N Engl J Med 378:1999-2009

41. Loomis D, Grosse Y, Lauby-Secretan B et al (2013) The carcinogenicity of outdoor air pollution. Lancet Oncol 14(13):1262

42. Beelen R, Hoek G, Brandt PA et al (2008) Long-term exposure to traffic-related air pollution and lung cancer risk. Epidemiol 19(5):702-710

43. Raaschou-Nielsen O, Bak H, Sørensen M et al (2010) Air pollution from traffic and risk for lung cancer in three Danish cohorts. Cancer Epidemiol Biomarkers Prev 19(5):1284-1291

44. Huang F, Pan B, Wu J et al (2017) Relationship between exposure to PM2.5 and lung cancer incidence and mortality: a metaanalysis. Oncotarget 8:43322-43331

Publisher's Note Springer Nature remains neutral with regard to jurisdictional claims in published maps and institutional affiliations. 